\title{
Unraveling trastuzumab and lapatinib inefficiency in gastric cancer: Future steps (Review)
}

\author{
SHOUJI SHIMOYAMA \\ Gastrointestinal Unit, Settlement Clinic, Tokyo 120-0003, Japan
}

Received August 25, 2013; Accepted November 19, 2013

DOI: $10.3892 / \mathrm{mco} .2013 .218$

\begin{abstract}
The newly developed concept of oncogene addiction provides a rationale for the use of targeted therapies. In sharp contrast to the field of breast cancer treatment, attempts to target human epidermal growth factor receptor 2 (HER2) among gastric cancer (GC) patients have been unsatisfactory. The ToGA trial reported only a modest prolongation of progression-free survival (PFS) with trastuzumab and the subsequent TYTAN and LOGiC trials failed to demonstrate any survival advantage with lapatinib. These results suggest that a response to the molecular-targeted therapies is achieved in only a fraction of the patients; in addition, even responders may experience secondary resistance, with the efficacy of the treatment being decreased or abrogated over a short period of time. Considering the increased recognition of primary or acquired resistance, recent investigations on targeted therapies have been primarily focused on determining in advance the mechanisms that may mediate resistance to treatment and the methods through which such obstacles may be circumvented. The proposed molecules or mechanisms that may be responsible for the development of resistance to single HER2-targeted therapy include a dimerization partner or crosstalk with HER2, such as HER3 and MET, as well as any subsequent activation of their downstream pathways, which exhibit a partial overlap with those of HER2. Furthermore, genetic alterations that stimulate the aberrant activation of the pathways downstream of HER2 may be the underlying mechanisms that restore prosurvival signaling. These mechanisms generate a complex signaling network with a significant potential for signal amplification and diversification. Although in the early stages of description, several compounds have been suggested as next generation treatments for GC, with expectations for their delineating the function of such receptors or molecules, with subsequent contributions of specific survival signaling blockades. This review focuses on the current achievements of anti-HER2 therapies in GC and the plausible mechanisms
\end{abstract}

Correspondence to: Dr Shouji Shimoyama, Gastrointestinal Unit, Settlement Clinic, 4-20-7 Towa, Adachi-ku, Tokyo 120-0003, Japan E-mail: shimoyama@apost.plala.or.jp

Key words: gastric cancer, human epidermal growth factor receptor, trastuzumab, lapatinib, resistance of resistance to these therapies. Elucidating these mechanisms of resistance may provide valuable information pertinent to the design of future strategies to improve molecular-targeted therapies.

\section{Contents}

1. Introduction

2. Current achievements with the clinical use of trastuzumab

3. Lapatinib is currently unsatisfactory

4. HER2 is an orphan receptor

5. Possible strategies to overcome de novo and acquired resistance to targeting HER2

6. Ongoing clinical trials

7. Future perspectives

\section{Introduction}

Gastric cancer (GC) is the fourth most commonly diagnosed cancer and the second leading cause of cancer-related mortality (1). GC is usually diagnosed at an advanced stage and patients with GC exhibit a poor prognosis. Overall 5-year survival rates are $\sim 30 \%$ (2) in the USA and are $<45 \%$ for stage III GC, even in Japan (3). In patients with advanced-stage disease, the median overall survival (OS) and median progression-free survival (PFS) were 9-13 and 6-7 months, respectively, even with the implementation of doublet (4) or triplet (5) therapeutic regimens. These results suggested that even the most widely accepted regimens, such as fluoropyrimidine and/or platinum-based chemotherapy regimens, have not been able to achieve satisfactory survival times. Thus, there is an urgent need to develop more efficient therapies.

Furthermore, conventional chemotherapy agents, which are predominantly effective on proliferating cells, often lack an effective chemotherapeutic window, as they are unable to discriminate between rapidly dividing normal cells (e.g., bone marrow and gastrointestinal tract cells) and tumor cells, leading to undesirable toxicities. Drug development in oncology aims for specificity in eliminating malignant cells with minimal side effects, thus creating a niche for targeted-driven approaches that are aimed at key molecules, which are overexpressed on tumors and are crucial for the development and spread of the disease. 
This conceptual shift focusing on key molecules has prompted interest in the development of molecular-targeted therapy. This type of therapy is based on the concept of oncogene addiction, i.e., certain cancers are apparently dependent on one or more genes for the maintenance of the malignant phenotype (6). This concept has led to the development of novel treatment strategies, where the removal or suppression of such a gene or genes or their coded molecules may specifically inhibit the growth of cancer cells, with resultant improved patient survival. Candidates for targeted molecules in the field of cancer research are members of membrane-bound human epidermal growth factor receptors (HERs), which consist of a ligand-binding domain on the extracellular surface, a single transmembrane segment and a cytoplasmic portion harboring the protein kinase activity. Among HER family members, such as human epidermal growth factor receptor (EGFR, also referred to as HER1), HER2, HER3 and HER4, HER2 has become the focus of investigations in GC.

The reported HER 2 amplification in $\sim 13-23 \%$ of GC cases (7) has prompted an investigation into the efficacy of targeting this molecule and the subsequent inhibition of the cell growth signaling cascade. HER2 overexpression is more frequent in intestinal compared with that in diffuse type GC and in cancers of the gastroesophageal junction compared with that in cancers located elsewhere in the stomach (7). As the overexpression of HER2 enables the constitutive activation of growth signaling pathways and thereby serves as an oncogenic driver, direct targeting of HER 2 and inhibition of the HER2-activated signal transduction is likely to provide therapeutic possibilities for HER2-positive GC patients. Therefore, HER2 has become an attractive target in clinical trials; however, its efficacy was shown to be modest, or less satisfactory than what would be expected (8-10). These unsatisfactory results may be attributed to primary (de novo) or secondary (acquired) resistance to the HER2-targeted therapy. Therefore, the focus of future investigations should be the development of treatment strategies to overcome this resistance, on the basis of a better understanding of the molecular, structural and biological properties of HER2 and/or other HER family members. The aim of this review was to summarise the current achievements in targeting HER2 and the possible mechanisms underlying the limited efficacy of the HER2 axis-targeting therapies in GC. Furthermore, we aimed to provide insights for the development of strategies that may ultimately overcome resistance to or limited efficacy of anti-HER2 axis treatments for GC.

\section{Current achievements with the clinical use of trastuzumab}

Trastuzumab is a humanized monoclonal anti-HER 2 antibody directed against the HER2 ectodomain. Although no specific ligands for HER2 have been identified (11), several extracellular and intracellular antitumor mechanisms of trastuzumab have been determined. These mechanisms include internalization and degradation of HER2 (12), downregulation of HER2 expression (13), inhibition of ligand-independent HER2 signaling (14), antibody-dependent cytotoxic activity (15), inhibition of p95HER2 formation, which is a constitutively active form of HER2 (16) and a predictor of unfavorable outcome (17), suppression of tumor angiogenesis (18) and inhibition of DNA damage repair (14).
Trastuzumab has been approved for the treatment of HER2-positive GC and a recent randomized ToGA trial (8) demonstrated that a combination of trastuzumab and chemotherapy achieved a clinical benefit compared to chemotherapy alone in terms of tumor response, PFS and OS. However, the absolute increase in the response rate with the addition of trastuzumab was only $12 \%$, with an overall response rate of $<50 \%$. Furthermore, the GC patients who achieved an initial therapeutic response to trastuzumab eventually exhibited disease progression within 7 months. Those findings suggested that a significant proportion of patients with HER2-positive GC either do not satisfactorily respond to trastuzumab or develop an acquired resistance to this antibody. Indeed, the tumor response to trastuzumab treatment in HER2-positive breast cancer patients was limited and the majority of those who initially responded to trastuzumab ultimately became resistant during prolonged treatment $(19,20)$. Those findings suggested that there may be a weakness in molecular-targeted therapies against single receptors, such as HER2, due to the potential activation of compensatory or overlapping survival pathways. Considering the increasing incidence in cancers of the gastroesophageal junction (21), in which HER2-overexpression is characteristic, the development of alternative strategies to overcome trastuzumab resistance is critical.

\section{Lapatinib is currently unsatisfactory}

Another molecule used for targeting HER2 is lapatinib. Lapatinib is a dual potent tyrosine kinase inhibitor that simultaneously curbs the phosphorylation of HER2 and EGFR, thus interrupting the EGFR/HER2-associated downstream signaling cascades. Theoretically, lapatinib and trastuzumab act synergistically, which was confirmed by the significantly improved PFS and OS in trastuzumab-refractory breast cancer patients undergoing lapatinib-trastuzumab combination treatment (22). Furthermore, lapatinib was also found to be effective against cancer cells expressing an aberrant form of HER2 (p95HER2), which may be one of the factors conferring trastuzumab resistance, as it lacks the extracellular domain of HER2 that is responsible for the impaired access of trastuzumab to the HER2 protein (23). A recent meta-analysis of randomized phase III trials clearly demonstrated the clinical benefits of lapatinib in the treatment of HER2-positive breast cancer patients (24).

With regard to GC, several preclinical studies demonstrated the antiproliferative effects of lapatinib (25), in addition to the synergic action of lapatinib with trastuzumab (26) or with other chemotherapeutic agents (25). Furthermore, lapatinib was shown to downregulate thymidylate synthase, which is a key determinant of fluoropyrimidine sensitivity, suggesting the potentiation of fluoropyrimidine efficacy (27). However, a phase II study on lapatinib as first-line treatment achieved only a $9 \%$ response rate, with a median OS of 4.8 months (28). Subsequently, a TYTAN study, comparing a combination of lapatinib and weekly paclitaxel to weekly paclitaxel alone as second-line treatment in HER2-positive GC, failed to demonstrate any OS and PFS benefit, although the median OS was prolonged by 2 months with lapatinib (9). Furthermore, the LOGiC phase III trial investigated the efficacy of lapatinib with capecitabine and 
oxaliplatin in gastric, esophageal and esophagogastric adenocarcinoma. The results of that trial were recently reported and demonstrated a non-significant prolongation (1.7 months) of OS with the addition of lapatinib (10).

The aforementioned limited efficacy of trastuzumab in the ToGA trial (8) and the unsatisfactory results of the TYTAN (9) and LOGiC trials (10) suggest the presence of drug resistance mechanisms or alternative pathways of escape from HER2-targeted therapy. To improve the treatment results of targeting HER2 in HER2-positive GC patients, there is an urgent need to elucidate the mechanisms underlying the alterations in tumor cell sensitivity to anti-HER2 therapy and to establish rational strategies to overcome this phenomenon. Therefore, the roles and functions of HER2 should be revisited and reconsidered.

\section{HER2 is an orphan receptor}

HER2 is a 185-kDa transmembrane receptor tyrosine kinase (29). Ligand-induced dimerization, with resultant autophosphorylation of their cytoplasmic domains, is a major mode of activation of receptor tyrosine kinases. In other HER family members, such as EGFR (HER1), HER3 and HER4, ligand binding to cognate HER includes conformational changes in the receptor, which subsequently promote homo- or heterodimerization with other HER family members. By contrast, no ligands of HER 2 have been identified (11). However, the overexpression of HER 2 in cell lines leads to transformation in the absence of a ligand (30), suggesting that HER2 is an orphan receptor. Instead, HER2 is a preferred dimerization partner and is poised for interaction with other HER family members; thus, HER2 mainly functions as a shared co-receptor (31).

As the only member of the family lacking an exogenous ligand,HER2 is transactivated through heterodimerization with all the other HER family members. The HER2 extracellular domain is always in the open conformation, leading to autoactivation (32) in addition to the formation of heterodimers with other HER family members, even when monomeric $(32,33)$, which may explain why HER2 is a preferred dimerization partner for other HER family members (31). Conversely, HER1 and HER3 cannot be transformed in the absence of a ligand (32). Notably, the HER2-containing heterodimers are the most potent complexes (34), leading to the activation of cascades in the downstream signaling transduction of the Ras/Raf/mitogen-activated protein kinase (MAPK) and phosphatidylinositol 3-kinase (PI3K)/Akt/mammalian target of rapamycin (mTOR) pathways (35). In HER2-positive GC cells, such pathways transmit signals promoting cell growth, differentiation, adhesion, migration and survival, contributing to tumor aggressiveness (35).

Among HER2-associated dimers, the HER2/HER3 heterodimer is considered to be the most active $(29,36,37)$, since HER2 has no known ligand and the intrinsic tyrosine kinase of HER3 is defective. Indeed, the overexpression of HER3 has been associated with worse survival rates in various types of cancer (37). Furthermore, in GC, HER2 and/or HER3 overexpression were found to be significant predictors of poor survival (38-40). Therefore, inhibiting HER2/HER3 heterodimerization and targeting molecules involved in HER2-associated signal amplification and diversification may be a viable strategy to potentiate the therapeutic activity of or overcome resistance to anti-HER2 therapy. There are several approaches to realize this potentiation, as described below.

\section{Possible strategies to overcome de novo and acquired resistance to targeting HER2}

Pertuzumab. In sharp contrast to trastuzumab, which binds to domain IV of HER2, pertuzumab is a monoclonal antibody that binds to domain II of HER2, the interface of dimer formation. As the HER2 domain IV is a region not involved in receptor dimerization (32), this may explain why HER ligands are able to induce the formation of HER2-containing heterodimers even in the presence of the trastuzumab, and why pertuzumab successfully inhibits the dimerization of HER2 with other HER family proteins (including HER3) and prevents ligand-dependent HER signaling (36). Previous in vitro studies have suggested that pertuzumab exhibits antitumor activity through HER2/HER3 signaling inhibition in non-small-cell lung (41) and breast cancer (42). A recent phase III trial (CLEOPATRA) demonstrated prolonged PFS (43) and OS (44) in metastatic breast cancer patients with the addition of pertuzumab to trastuzumab plus docetaxel, compared to trastuzumab plus docetaxel alone. A subsequent review demonstrated the clinical benefits of pertuzumab in several types of cancer (36).

The number of available studies on pertuzumab in GC, even in preclinical models, is limited. A HER2-positive GC xenograft model consistently demonstrated enhanced antitumor activity with the combination of pertuzumab and trastuzumab (45).

Targeting HER3. HER3 cannot form homodimers and only forms heterodimers that are required for activating signaling pathways (46). HER3 has been disregarded as a cancer target due to the absence of intrinsic kinase activity in its intracellular domain. However, HER3 is currently recognized as one of the key molecules associated with resistance to HER2-targeted therapies. HER3 was shown to potently activate the PI3K/Akt signaling pathway, despite its lack of intrinsic kinase activity, through its six docking sites for the p85 subunit of PI3K $(47,48)$, which allow HER3 to funnel its signals as a scaffold protein and, consequently, to evade the actions of trastuzumab or HER-associated tyrosine kinase inhibitors. Therefore, the upregulated activity of HER3 may be a means of escape from therapeutic suppression by anti-HER2 agents, rendering currently available anti-HER2 therapies considerably less effective. Therefore, the HER2/HER3 heterodimer is considered to be a functional HER signaling unit. For example, upregulation of HER3 was observed in trastuzumab-resistant breast cancer cells following long-term exposure to trastuzumab (49). Furthermore, preferential phosphorylation of HER3 was observed in HER2-amplified breast cancer tissues (42). Furthermore, the loss of HER3 compromised the viability of HER2-overexpressing breast cancer cells (50). An in vitro study on HER2-positive breast cancer cells demonstrated a PI3K/Akt-dependent upregulation of HER3 mRNA following inhibition of HER2 tyrosine kinase with lapatinib, suggesting a HER3-mediated compensation pathway in the presence of HER2 inhibition (51). Indeed, EGFR and HER2 
Table I. Ongoing clinical trials investigating molecules that may overcome de novo or acquired resistance to trastuzumab in gastric cancer.

\begin{tabular}{|c|c|c|c|c|c|}
\hline Molecules & Trial number & Conditions & Combined agents & Phase & Refs. \\
\hline \multirow[t]{2}{*}{ Afatinib $^{a}$} & NCT01649271 & HER2-positive gastric or breast cancer & Trastuzumab & Phase I & $(53,78)$ \\
\hline & NCT01522768 & $\begin{array}{l}\text { HER2-positive, trastuzumab-refractory } \\
\text { esophagogastric cancer }\end{array}$ & None & Phase II & \\
\hline LJM716 $^{\mathrm{b}}$ & NCT01602406 & $\begin{array}{l}\text { HER2-positive, trastuzumab-refractory } \\
\text { gastric or breast cancer }\end{array}$ & Trastuzumab & Phase I & (79) \\
\hline MK-2206 & NCT01705340 & $\begin{array}{l}\text { HER2-positive breast, gastric, } \\
\text { or gastroesophageal cancer }\end{array}$ & Trastuzumab, lapatinib & Phase I & $(80)$ \\
\hline MM-111 & NCT01774851 & $\begin{array}{l}\text { HER2-positive distal esophageal, } \\
\text { gastric, or gastroesophageal cancer }\end{array}$ & Trastuzumab, paclitaxel & $\begin{array}{l}\text { Randomized } \\
\text { phase II }\end{array}$ & $(81)$ \\
\hline \multirow[t]{2}{*}{ Pertuzumab $^{\mathrm{e}}$} & NCT01461057 & $\begin{array}{l}\text { HER2-positive gastric or } \\
\text { gastroesophageal cancer }\end{array}$ & $\begin{array}{l}\text { Trastuzumab, cisplatin, } \\
\text { capecitabine }\end{array}$ & Phase II & (36) \\
\hline & NCT01774786 & $\begin{array}{l}\text { HER2-positive gastric or } \\
\text { gastroesophageal cancer }\end{array}$ & $\begin{array}{l}\text { Trastuzumab, cisplatin, } \\
\text { capecitabine (or } \\
\text { 5-fluorouracil) }\end{array}$ & Phase III & \\
\hline Poziotinib $^{f}$ & NCT01746771 & $\begin{array}{l}\text { HER2-positive gastric or } \\
\text { gastroesophageal cancer }\end{array}$ & Trastuzumab, paclitaxel & Phase I/II & $(82)$ \\
\hline
\end{tabular}

${ }^{a}$ Inhibitor of all HER family receptor tyrosine kinases. ${ }^{\mathrm{b}}$ Anti-HER3 antibody. ${ }^{\mathrm{c}}$ Akt inhibitor. ${ }^{\mathrm{d} B i s p e c i f i c}$ antibody that targets HER2 and HER3. ${ }^{\mathrm{e}}$ Anti-HER2 domain II. ${ }^{\mathrm{f}}$ Pan-HER inhibitor. HER, human epidermal growth factor receptor.

targeting was succeeded by a feedback upregulation of HER3 (52). Those findings suggested that the HER3-PI3K axis may be essential for the survival of HER2-dependent cells and the ligand-less HER2 and kinase-dead HER3 may form a formidable molecule when dimerized. Since HER3 may weaken the action of anti-HER 2 therapy, the combined targeting of HER2 and HER3 may act synergistically on HER2 and HER3 downstream signaling pathways and may be of therapeutic value. In GC, this hypothesis was confirmed in a preclinical study, in which a pan-HER inhibitor was shown to act synergistically with trastuzumab (53).

Inhibition of MET. A functional crosstalk between MET and HER family members has been reported in the context of the acquisition of invasive and progressive phenotypes (54-56). The receptor tyrosine kinase MET stimulates cell proliferation and invasion via the activation of the MAPK and the PI3K/Akt axis, which are also involved in the downstream signaling cascade of the HER family (57). For example, gefitinib-sensitive lung cancer cell lines reportedly developed resistance to gefitinib as a result of the amplification of the MET gene and the subsequent HER3-dependent PI3K activation (58). Similarly, MET contributed to evoking resistance to anti-EGFR therapy in colorectal cancer (59) and to anti-HER2 therapy in breast cancer (60). Those findings suggested that MET may be involved in the compensation for HER family inhibition.

With regard to $\mathrm{GC}$, amplification of the MET gene and overexpression of the MET protein were observed in 10-20 and 50\% of tumors, respectively (61-63). The hepatocyte growth factor-mediated activation of MET may also mediate resistance to lapatinib in HER2-amplified GC cell lines by restimulating downstream signaling (64). It was demonstrated that MET and HER family members form an extensive network of downstream signaling in the MKN-45 GC cell line (65). Furthermore, the MET-independent reactivation of the MAPK or PI3K pathways may alleviate the growth-inhibiting effects of MET kinase inhibition (66). Those findings suggested that the MET and HER families may cooperate, in the sense that inhibition of one receptor may drive cells to activate alernative oncogenic signaling pathways. These mechanisms provide a rationale for the use of MET inhibitors to overcome resistance to HER2 axis inhibition. However, one should bear in mind that HER3 may also play a significant role in the acquisition of resistance to MET inhibition. GC cells resistant to MET inhibitors were shown to overexpress HER3 and HER3 silencing led to a reversion of the resistance to MET inhibitors, suggesting that the overexpression of HER3 may contribute to the development of resistance to MET inhibitors (67).

Targeting molecules downstream of HER2. Since the $\mathrm{PI} 3 \mathrm{~K} / \mathrm{Akt} / \mathrm{mTOR}$ cascade is a pathway downstream of HER2 and phosphatase and tensin homolog (PTEN) antagonizes the function of PI3K, the constitutive activation of PI3K occurs via two mechanisms: loss of PTEN function or activation of mutations in the gene encoding the catalytic PI3K subunit p110 $\alpha$ (PIK3CA). In breast cancer, the decreased expression of PTEN was found to be significantly correlated with a lower response to trastuzumab (68), whereas the introduction of antisense PTEN was associated with decreased sensitivity to trastuzumab (69), suggesting that PTEN deficiency may confer resistance to trastuzumab. Furthermore, PI3K inhibitors were shown to overcome trastuzumab resistance in PTEN-deficient breast cancer cells (68). PIK3CA activates Akt signaling, induces cell transformation (70), attenuates apoptosis and facilitates tumor invasion (71). Since gain-of-function muta- 
tions in PIK3CA may be involved in modulating the efficacy of HER2-directed therapies, the inhibition of Akt signaling effectively suppresses the growth of cancer cells that are dependent on this pathway (72). Indeed, PIK3CA mutations have been associated with shorter PFS after trastuzumab therapy (73). Those results suggested that trastuzumab resistance due to the aberrant activation of PI3K/Akt/mTOR signaling may be resolved by the inhibition of this pathway.

In GC, PIK3CA amplification was observed in 36\% of GC tissues and $60 \%$ of GC cell lines and was associated with Akt activation (74). Since mutations and loss of heterozygosity of PTEN were observed in 10 and $17 \%$ of GC, respectively $(75,76)$, inhibitors of the PI3K/Akt/mTOR pathway activated by PTEN loss may resolve de novo and acquired non-responsiveness to trastuzumab. Notably, mutations of PTEN and PIK3CA are mutually exclusive (74). This may explain why PTEN loss or PIK3CA mutations were not individually sufficient to predict a diminished response to trastuzumab-based therapy, whereas their combinations were (77). Elucidating the genetically defined response mechanisms may indicate the optimal target therapy for each patient.

\section{Ongoing clinical trials}

Numerous molecules designed to overcome resistance to anti-HER2 therapy were introduced over the last few years. Table I lists the ongoing clinical trials investigating novel molecules that may help overcome primary and acquired trastuzumab resistance in GC. These molecules include afatinib [irreversible inhibitor of all HER family receptor tyrosine kinases $(53,78)]$, LJM716 [anti-HER3 antibody (79)], MK-2206 [Akt inhibitor (80)], MM-111 [bispecific antibody that targets HER2 and HER3 (81)], pertuzumab and poziotinib [pan-HER inhibitor (82)]. These molecules are administered alone or in combination with trastuzumab and/or lapatinib, with or without concurrent chemotherapy.

\section{Future perspectives}

Anti-HER2 therapy, such as trastuzumab and lapatinib, accrues clinical benefit in certain GC patients; however, a number of patients exhibit primary resistance and those who initially respond to treatment may eventually develop secondary resistance, suggesting that other factors that involve resistance or hinder response should be considered prior to treatment or at the time of resistance development. Two major categories of resistance mechanisms to anti-HER2 therapy have been proposed: de novo resistance, due to genetic alterations of HER2 and its downstream signaling targets; and acquired resistance, primarily due to alternative pathway activation to compensate for HER2 inhibition.

Although the precise mechanisms underlying therapeutic responsiveness have not been fully elucidated, even in oncogene-addicted tumors, it is essential to understand that the targeting of one receptor may be neutralized or attenuated by the parallel activation of other receptors, molecules and pathways. Such an orchestration bestows resistance on cancer cells, helping them escape from targeted therapies. For example, the signal elicited by a heterodimerized receptor is not simply the sum of the signaling properties of the individual dimerization partners. The complexity of these plausible resistance mechanisms underlines the potential limitations of monotherapy and should prompt a conceptual change from inhibiting only one molecule to a combined oncogenic pathway inhibition. Therefore, it is advisable to employ combinations of targeted therapies that may delineate more than single HER2-associated biological outcomes, thereby yielding maximal therapeutic benefit.

The key goals of future studies in the research field of GC treatment should include the development of novel therapeutics that overcome resistance to anti-HER2 therapy effectively and safely, with the identification of accurate predictors of response. For these purposes, certain compounds have already entered clinical development, for the targeting of other receptors or molecules synergizing with HER2. Accordingly, it was attempted to use these compounds in combination with current anti-HER2 therapy, rather than as single agents. Although clinical proof of the concept behind these agents has not yet been provided, this is anticipated in the immediate future and may further accelerate the inclusion of such inhibitors in combination treatments, as part of a repertoire of more effective anticancer agents. Parallel efforts should also be focused on identifying suitable biomarkers for the selection of the optimal combination agents and the patients who are most likely to benefit from such treatment. Ideally, the predictive biomarkers for targeted therapy should be identified by feasible and reliable assays, so that they are applicable to other targeted therapies.

Considering that oncogene addiction is commonly observed in several types of cancer, the mechanisms of abnormal interplay between molecules involved in survival cascades, as well as novel agents that effectively inhibit the processes identified in a specific type of cancer, may also be applied to other types of cancer. This approach may provide more flexibility in terms of targeting resistance mediators in one type of cancer to be applicable to other cancers that share common resistance mechanisms. Expanding the understanding of resistance mechanisms from 'each-organ based' to a 'trans-organ basis' may facilitate the establishment of more global treatment strategies against tumors addicted to a common oncogenic pathway, rather than against individual tumors. We are confident that such joint efforts, i.e., the continued exchange of information between the clinical fields of each cancer, or the translation of the findings from preclinical studies into the clinical realm, will uncover novel agents that underlie clinical responses and overcome resistance to molecular-targeted therapy. This may help establish effective treatment strategies rationally rather than empirically. These novel agents, once verified and validated, may become the next 'gold standard' among cancer therapies and, if combined with reliable biomarkers for resistance and response prediction, may ultimately introduce a new era of personalized medicine.

\section{References}

1. Jemal A, Center MM, DeSantis C and Ward EM: Global patterns of cancer incidence and mortality rates and trends. Cancer Epidemiol Biomarkers Prev 19: 1893-1907, 2010.

2. Siegel R, Naishadham D and Jemal A: Cancer statistics, 2013. CA Cancer J Clin 63: 11-30, 2013.

3. Nashimoto A, Akazawa K, Isobe Y, et al: Gastric cancer treated in 2002 in Japan: 2009 annual report of the JGCA nationwide registry. Gastric Cancer 16: 1-27, 2013. 
4. Koizumi W, Narahara H, Hara T, et al: S-1 plus cisplatin versus S-1 alone for first-line treatment of advanced gastric cancer (SPIRITS trial): a phase III trial. Lancet Oncol 9: 215-221, 2008.

5. Cunningham D, Starling N, Rao S, et al: Capecitabine and oxaliplatin for advanced esophagogastric cancer. N Engl J Med 358: 36-46, 2008

6. Weinstein IB and Joe AK: Mechanisms of disease: Oncogene addiction - a rationale for molecular targeting in cancer therapy. Nat Clin Pract Oncol 3: 448-457, 2006.

7. Gravalos C and Jimeno A: HER2 in gastric cancer: a new prognostic factor and a novel therapeutic target. Ann Oncol 19 1523-1529, 2008.

8. Bang YJ, Van Cutsem E, Feyereislova A, et al; ToGA Trial Investigators: Trastuzumab in combination with chemotherapy versus chemotherapy alone for treatment of HER2-positive advanced gastric or gastro-oesophageal junction cancer (ToGA) a phase 3, open-label, randomised controlled trial. Lancet 376: 687-697, 2010.

9. Bang YJ: A randomized, open-label, phase III study of lapatinib in combination with weekly paclitaxel versus weekly paclitaxel alone in the second-line treatment of HER2 amplified advanced gastric cancer (AGC) in Asian population: Tytan study. J Clin Oncol 30 (Suppl 34): abstract 11, 2012.

10. Hecht JR, Bang YJ, Qin S, et al: Lapatinib in combination with capecitabine plus oxaliplatin (CapeOx) in HER2-positive advanced or metastatic gastric, esophageal, or gastroesophageal adenocarcinoma (AC): The TRIO-013/LOGiC Trial. J Clin Oncol 31 (Suppl): abstract LBA4001, 2013.

11. Olayioye MA, Neve RM, Lane HA and Hynes NE: The ErbB signaling network: receptor heterodimerization in development and cancer. EMBO J 19: 3159-3167, 2000.

12. Rubin I and Yarden Y: The basic biology of HER2. Ann Oncol 12 (Suppl 1): S3-S8, 2001.

13. Cuello M, Ettenberg SA, Clark AS, et al: Down-regulation of the erbB-2 receptor by trastuzumab (herceptin) enhances tumor necrosis factor-related apoptosis-inducing ligand-mediated apoptosis in breast and ovarian cancer cell lines that overexpress erbB-2. Cancer Res 61: 4892-4900, 2001.

14. Spector NL and Blackwell KL: Understanding the mechanisms behind trastuzumab therapy for human epidermal growth factor receptor 2-positive breast cancer. J Clin Oncol 27: 5838-5847, 2009.

15. Barok M, Isola J, Palyi-Krekk Z, et al: Trastuzumab causes antibody-dependent cellular cytotoxicity-mediated growth inhibition of submacroscopic JIMT-1 breast cancer xenografts despite intrinsic drug resistance. Mol Cancer Ther 6: 2065-2072, 2007.

16. Molina MA, Codony-Servat J, Albanell J, et al: Trastuzumab (herceptin), a humanized anti-Her2 receptor monoclonal antibody, inhibits basal and activated Her2 ectodomain cleavage in breast cancer cells. Cancer Res 61: 4744-4749, 2001.

17. Saez R, Molina MA, Ramsey EE, et al: p95HER-2 predicts worse outcome in patients with HER-2-positive breast cancer. Clin Cancer Res 12: 424-431, 2006.

18. Izumi $\mathrm{Y}, \mathrm{Xu} \mathrm{L}$, di Tomaso E, Fukumura D and Jain RK Tumour biology: herceptin acts as an anti-angiogenic cocktail. Nature 416: 279-280, 2002.

19. Vogel CL, Cobleigh MA, Tripathy D, et al: Efficacy and safety of trastuzumab as a single agent in first-line treatment of HER2-overexpressing metastatic breast cancer. J Clin Oncol 20 : 719-726, 2002.

20. Cobleigh MA, Vogel CL, Tripathy D, et al: Multinational study of the efficacy and safety of humanized anti-HER 2 monoclonal antibody in women who have HER2-overexpressing metastatic breast cancer that has progressed after chemotherapy for metastatic disease. J Clin Oncol 17: 2639-2648, 1999.

21. Kelley JR and Duggan JM: Gastric cancer epidemiology and risk factors. J Clin Epidemiol 56: 1-9, 2003.

22. Blackwell KL, Burstein HJ, Storniolo AM, et al: Overall survival benefit with lapatinib in combination with trastuzumab for patients with human epidermal growth factor receptor 2-positive metastatic breast cancer: final results from the EGF104900 study. J Clin Oncol 30: 2585-2592, 2012

23. Scaltriti M, Rojo F, Ocana A, et al: Expression of p95HER2, a truncated form of the HER 2 receptor, and response to anti-HER2 therapies in breast cancer. J Natl Cancer Inst 99: 628-638, 2007.

24. Amir E, Ocana A, Seruga B, Freedman O and Clemons M: Lapatinib and HER2 status: results of a meta-analysis of randomized phase III trials in metastatic breast cancer. Cancer Treat Rev 36: 410-415, 2010.
25. Kim JW, Kim HP, Im SA, et al: The growth inhibitory effect of lapatinib, a dual inhibitor of EGFR and HER2 tyrosine kinase, in gastric cancer cell lines. Cancer Lett 272: 296-306, 2008.

26. Wainberg ZA, Anghel A, Desai AJ, et al: Lapatinib, a dual EGFR and HER2 kinase inhibitor, selectively inhibits HER2-amplified human gastric cancer cells and is synergistic with trastuzumab in vitro and in vivo. Clin Cancer Res 16: 1509-1519, 2010.

27. Kim HP, Yoon YK, Kim JW, et al: Lapatinib, a dual EGFR and HER 2 tyrosine kinase inhibitor, downregulates thymidylate synthase by inhibiting the nuclear translocation of EGFR and HER2. PLoS One 4: e5933, 2009.

28. Iqbal S, Goldman B, Fenoglio-Preiser CM, et al: Southwest Oncology Group study S0413: a phase II trial of lapatinib (GW572016) as first-line therapy in patients with advanced or metastatic gastric cancer. Ann Oncol 22: 2610-2615, 2011.

29. Akiyama T, Sudo C, Ogawara H, Toyoshima K and Yamamoto T: The product of the human c-erbB-2 gene: a 185-kilodalton glycoprotein with tyrosine kinase activity. Science 232: 1644-1646, 1986

30. Di Fiore PP, Pierce JH, Kraus MH, et al: erbB-2 is a potent oncogene when overexpressed in NIH/3T3 cells. Science 237: $178-182,1987$.

31. Graus-Porta D, Beerli RR, Daly JM and Hynes NE: ErbB-2, the preferred heterodimerization partner of all ErbB receptors, is a mediator of lateral signaling. EMBO J 16: 1647-1655, 1997.

32. Cho HS, Mason K, Ramyar KX, et al: Structure of the extracellular region of HER2 alone and in complex with the Herceptin Fab. Nature 421: 756-760, 2003.

33. Garrett TP, McKern NM, Lou M, et al: The crystal structure of a truncated ErbB2 ectodomain reveals an active conformation, poised to interact with other ErbB receptors. Mol Cell 11: 495-505, 2003

34. Yarden Y and Sliwkowski MX: Untangling the ErbB signalling network. Nat Rev Mol Cell Biol 2: 127-137, 2001.

35. Wong $\mathrm{H}$ and Yau T: Targeted therapy in the management of advanced gastric cancer: are we making progress in the era of personalized medicine? Oncologist 17: 346-358, 2012.

36. Baselga J and Swain SM: Novel anticancer targets: revisiting ERBB2 and discovering ERBB3. Nat Rev Cancer 9: 463-475, 2009.

37. Ocana A, Vera-Badillo F, Seruga B, et al: HER3 overexpression and survival in solid tumors: a meta-analysis. J Natl Cancer Inst 105: 266-273, 2013.

38. Hayashi M, Inokuchi M, Takagi Y, et al: High expression of HER3 is associated with a decreased survival in gastric cancer. Clin Cancer Res 14: 7843-7849, 2008.

39. Begnami MD, Fukuda E, Fregnani JH, et al: Prognostic implications of altered human epidermal growth factor receptors (HERs) in gastric carcinomas: HER2 and HER3 are predictors of poor outcome. J Clin Oncol 29: 3030-3036, 2011.

40. Zhang XL, Yang YS, Xu DP, et al: Comparative study on overexpression of HER $2 /$ neu and HER3 in gastric cancer. World J Surg 33. 2112-2118, 2009

41. Sakai K, Yokote H, Murakami-Murofushi K, Tamura T, Saijo N and Nishio K: Pertuzumab, a novel HER dimerization inhibitor, inhibits the growth of human lung cancer cells mediated by the HER3 signaling pathway. Cancer Sci 98: 1498-1503, 2007.

42. Lee-Hoeflich ST, Crocker L, Yao E, Pham T, Munroe X, Hoeflich KP, Sliwkowski MX and Stern HM: A central role for HER3 in HER2-amplified breast cancer: implications for targeted therapy. Cancer Res 68: 5878-5887, 2008.

43. Baselga J, Cortes J, Kim SB, et al: Pertuzumab plus trastuzumab plus docetaxel for metastatic breast cancer. N Engl J Med 366: 109-119, 2012

44. Swain SM, Kim SB, Cortes J, et al: Pertuzumab, trastuzumab, and docetaxel for HER2-positive metastatic breast cancer (CLEOPATRA study): overall survival results from a randomised, double-blind, placebo-controlled, phase 3 study. Lancet Oncol 14: 461-471, 2013.

45. Yamashita-Kashima Y, Iijima S, Yorozu K, Furugaki K, Kurasawa M, Ohta M and Fujimoto-Ouchi K: Pertuzumab in combination with trastuzumab shows significantly enhanced antitumor activity in HER2-positive human gastric cancer xenograft models. Clin Cancer Res 17: 5060-5070, 2011

46. Berger MB, Mendrola JM and Lemmon MA: ErbB3/HER3 does not homodimerize upon neuregulin binding at the cell surface. FEBS Lett 569: 332-336, 2004.

47. Schulze WX, Deng L and Mann M: Phosphotyrosine interactome of the ErbB-receptor kinase family. Mol Syst Biol 1: 2005.0008, 2005.

48. Hsieh AC and Moasser MM: Targeting HER proteins in cancer therapy and the role of the non-target HER3. Br J Cancer 97: $453-457,2007$ 
49. Narayan M, Wilken JA, Harris LN, Baron AT, Kimbler KD and Maihle NJ: Trastuzumab-induced HER reprogramming in 'resistant' breast carcinoma cells. Cancer Res 69: 2191-2194, 2009.

50. Holbro T, Beerli RR, Maurer F, Koziczak M, Barbas CF III and Hynes NE: The ErbB2/ErbB3 heterodimer functions as an oncogenic unit: ErbB2 requires ErbB3 to drive breast tumor cell proliferation. Proc Natl Acad Sci USA 100: 8933-8938, 2003.

51. Garrett JT, Olivares MG, Rinehart C, et al: Transcriptional and posttranslational up-regulation of HER3 (ErbB3) compensates for inhibition of the HER2 tyrosine kinase. Proc Natl Acad Sci USA 108: 5021-5026, 2011.

52. Sergina NV, Rausch M, Wang D, Blair J, Hann B, Shokat KM and Moasser MM: Escape from HER-family tyrosine kinase inhibitor therapy by the kinase-inactive HER3. Nature 445 : 437-441, 2007.

53. Nam HJ, Ching KA, Kan J, et al: Evaluation of the antitumor effects and mechanisms of PF00299804, a pan-HER inhibitor alone or in combination with chemotherapy or targeted agents in gastric cancer. Mol Cancer Ther 11: 439-451, 2012.

54. Khoury H, Naujokas MA, Zuo D, et al: HGF converts ErbB2/Neu epithelial morphogenesis to cell invasion. Mol Biol Cell 16 : 550-561, 2005

55. Lai AZ, Abella JV and Park M: Crosstalk in Met receptor oncogenesis. Trends Cell Biol 19: 542-551, 2009.

56. Puri N and Salgia R: Synergism of EGFR and c-Met pathways, cross-talk and inhibition, in non-small cell lung cancer. J Carcinog 7: 9, 2008.

57. Trusolino L, Bertotti A and Comoglio PM: MET signalling: principles and functions in development, organ regeneration and cancer. Nat Rev Mol Cell Biol 11: 834-848, 2010.

58. Engelman JA, Zejnullahu K, Mitsudomi T, et al: MET amplification leads to gefitinib resistance in lung cancer by activating ERBB3 signaling. Science 316: 1039-1043, 2007.

59. Liska D, Chen CT, Bachleitner-Hofmann T, Christensen JG and Weiser MR: HGF rescues colorectal cancer cells from EGFR inhibition via MET activation. Clin Cancer Res 17: 472-482, 2011

60. Shattuck DL, Miller JK, Carraway KL III and Sweeney C: Met receptor contributes to trastuzumab resistance of Her2-overexpressing breast cancer cells. Cancer Res 68 1471-1477, 2008

61. Nakajima M, Sawada H, Yamada Y, et al: The prognostic significance of amplification and overexpression of c-met and c-erb B-2 in human gastric carcinomas. Cancer 85: 1894-1902, 1999.

62. Kuniyasu H, Yasui W, Kitadai Y, Yokozaki H, Ito $\mathrm{H}$ and Tahara E: Frequent amplification of the c-met gene in scirrhous type stomach cancer. Biochem Biophys Res Commun 189 227-232, 1992.

63. Janjigian YY, Tang LH, Coit DG, et al: MET expression and amplification in patients with localized gastric cancer. Cancer Epidemiol Biomarkers Prev 20: 1021-1027, 2011

64. Chen CT, Kim H, Liska D, Gao S, Christensen JG and Weiser MR: MET activation mediates resistance to lapatinib inhibition of HER2-amplified gastric cancer cells. Mol Cancer Ther 11: 660-669, 2012.

65. Guo A, Villén J, Kornhauser J, et al: Signaling networks assembled by oncogenic EGFR and c-Met. Proc Natl Acad Sci USA 105: 692-697, 2008.

66. Bachleitner-Hofmann T, Sun MY, Chen CT, et al: HER kinase activation confers resistance to MET tyrosine kinase inhibition in MET oncogene-addicted gastric cancer cells. Mol Cancer Ther 7: 3499-3508, 2008.
67. Corso S, Ghiso E, Cepero V, et al: Activation of HER family members in gastric carcinoma cells mediates resistance to MET inhibition. Mol Cancer 9: 121, 2010.

68. Nagata Y, Lan KH, Zhou X, et al: PTEN activation contributes to tumor inhibition by trastuzumab, and loss of PTEN predicts trastuzumab resistance in patients. Cancer Cell 6: 117-127, 2004

69. Fujita T, Doihara H, Kawasaki K, et al: PTEN activity could be a predictive marker of trastuzumab efficacy in the treatment of ErbB2-overexpressing breast cancer. Br J Cancer 94: 247-252, 2006.

70. Isakoff SJ, Engelman JA, Irie HY, et al: Breast cancer-associated PIK3CA mutations are oncogenic in mammary epithelial cells. Cancer Res 65: 10992-11000, 2005.

71. Samuels Y, Diaz LA Jr, Schmidt-Kittler O, et al: Mutant PIK3CA promotes cell growth and invasion of human cancer cells. Cancer Cell 7: 561-573, 2005

72. She QB, Chandarlapaty S, Ye Q, et al: Breast tumor cells with PI3K mutation or HER2 amplification are selectively addicted to Akt signaling. PLoS One 3: e3065, 2008.

73. Berns K, Horlings HM, Hennessy BT, et al: A functional genetic approach identifies the PI3K pathway as a major determinant of trastuzumab resistance in breast cancer. Cancer Cell 12: 395-402, 2007.

74. Byun DS, Cho K, Ryu BK, et al: Frequent monoallelic deletion of PTEN and its reciprocal association with PIK3CA amplification in gastric carcinoma. Int J Cancer 104: 318-327, 2003.

75. Oki E, Kakeji Y, Baba H, et al: Impact of loss of heterozygosity of encoding phosphate and tensin homolog on the prognosis of gastric cancer. J Gastroenterol Hepatol 21: 814-818, 2006.

76. Li YL, Tian Z, Wu DY, Fu BY and Xin Y: Loss of heterozygosity on 10q23.3 and mutation of tumor suppressor gene PTEN in gastric cancer and precancerous lesions. World J Gastroenterol 11: 285-288, 2005.

77. Esteva FJ, Guo H, Zhang S, et al: PTEN, PIK3CA, p-AKT, and p-p70S6K status: association with trastuzumab response and survival in patients with HER2-positive metastatic breast cancer. Am J Pathol 177: 1647-1656, 2010.

78. Chen X, Zhu Q, Zhu L, et al: Clinical perspective of afatinib in non-small cell lung cancer. Lung Cancer 81: 155-161, 2013.

79. Garner A, Sheng Q, Bialucha U, et al: LJM716: an anti-HER3 antibody that inhibits both HER 2 and NRG driven tumor growth by trapping HER3 in the inactive conformation. Cancer Res 72 (Suppl 8): abstract 2733, 2012.

80. Hirai H, Sootome H, Nakatsuru Y, et al: MK-2206, an allosteric Akt inhibitor, enhances antitumor efficacy by standard chemotherapeutic agents or molecular targeted drugs in vitro and in vivo. Mol Cancer Ther 9: 1956-1967, 2010.

81. McDonagh CF, Huhalov A, Harms BD, et al: Antitumor activity of a novel bispecific antibody that targets the ErbB2/ErbB3 oncogenic unit and inhibits heregulin-induced activation of ErbB3. Mol Cancer Ther 11: 582-593, 2012.

82. Berardi R, Santoni M, Morgese F, et al: Novel small molecule EGFR inhibitors as candidate drugs in non-small cell lung cancer. Onco Targets Ther 6: 563-576, 2013. 